\title{
A Feature-Based Tool-Selection Classification for Agile Software Development
}

\author{
Mohsen Taheri \\ School of Computing and Information Sciences \\ Florida International University \\ Miami, FL 33199, USA \\ mtahe006@fiu.edu
}

\author{
S. Masoud Sadjadi \\ School of Computing and Information Sciences \\ Florida International University \\ Miami, FL 33199, USA \\ sadjadi@cs.fiu.edu
}

\begin{abstract}
With the advancement in technology, software development complexities are rising across the globe. This trend is forcing companies and organizations to adopt management methods and tools to accelerate time to market, more easily manage changing priorities, increase the customer satisfaction and reduce product expenses. Agile software development methods offer a solution to these issues, but problems remain over evaluation along with the offering of the correct agile software as well as a collection of agile tools. The purpose of this paper is to introduce best tools and features, criteria used for evaluating currently existing tools and propose a classification model to right agile tool selection. To prepare a list of the best tools and their features in the market, a practical research on existing tools and their features were performed. Finally, a classification model was prepared and the results show which tools best fit into different level of maturity in projects and companies.
\end{abstract}

Keywords-software development; agile tools; agile tool selection; feature-based classification.

\section{INTRODUCTION}

Agile software development is a set of software development methodologies based on incremental and iterative development in which specifications and alternatives, develop by means of cooperation between self-organizing, cross-functional groups. It promotes adaptive arranging, evolutionary improvement, early delivery, ongoing enhancement, and encourages rapid and accommodating response to change. In recent years, many startups, software companies and organizations adopting agile development methodology. They want to develop fast and high quality software products. Also, some other benefits obtained from implementing agile consist of the ability to deal with the software development visibility, cost, risk and priority management, to improve team moral and to make simpler project implementation process. This research is based on standard agile definitions and concepts and uses agile principles and agile manifesto to review the tools and their features. According to the agile manifesto, "individuals and interactions are over processes and tools, working software is over comprehensive documentation, customer collaboration is over contract negotiation and responding to change is over following a plan".

The market industry regarding software agile tools is now becoming more mature with commercial tools and dozens of small and large vendors which guide you to learn and work with agile methodology. Sometimes companies make mistake to choose appropriate tool, therefore many corporations arise three questions in their mind. First of all, which agile tools in the market is the best? Secondly, which agile tool is the best for our organization? And last but not least, how to select the right tool? Thus does a special agile tool fully meet all company expectations as a "one size fits all" tool for a product team, and make their collaboration and project tracking overall enjoyable.

Although there are many apps and tools offering traditional project management, tasks management \& To-Do List planning, this survey focuses only on agile project management tools, their specification and a classification to select best and right agile tool for each organization. This paper is organized as follows: Section 2 presents the literature review and previous works. Section 3 presents the methodology and research steps conducted during the study, agile tools, and describes the criteria used for evaluating currently existing tools. Section 4 analyses the lists and Section 5 presents the tool evaluation results. Finally, Section 6 concludes with final remarks $[1,2]$.

\section{LITERATURE REVIEW \& RELATED WORKS}

For this step, we analyzed the present white papers, journal and conference papers and best tool usage surveys in the agile development context. We went through many most important world's largest scientific and educational sources such as IEEE, ACM, Springer, Google scholar, and etc. We even surfed through less scientific online sources such as websites, whitepapers and published surveys. Finally, we only found few different surveys, which some of them were sponsored by tool vendors themselves. Some of the most relevant works to our research are presented as follows.

In 2011 Azizyan provides a list of features that are most desired by the existing software companies. Its result shows that the most satisfactory tool attribute is ease of use. As a positive point it is an unbiased survey and the negative point is it has focused just on gathering statistics as other surveys. Another negative point is the use of spreadsheets, yahoo groups, and the like to collect information using questionnaires. Although they had an IP tracker that it isn't reliable to have a normal distribution of countries, people and companies. This paper helps us to prepare a list of tools, criteria and metrics for our tables [3]. 
In 2012 Azizyan presents a journey towards agile tool selection for a specific anonymous company and the tool selection process is based on a study of the tool no functional features such as flexibility and usability. This paper gives a brief description of the company, then another section lists and describes the metrics used for evaluating currently existing tools. It has focused just on a special company and few tools, but in comparison with other papers, it introduces a methodology to select the right tool [4].

In 2006 “Agile Project Management (APM), Tooling Survey Results" focused on collecting statistics on tools used in requirements management, and also there are some statistics on agile method used and reasons for selecting an agile project management tool. It helps us to prepare a list of tools, criteria and metrics for our tables [5].

In 2008 "Agile tools: the good, the bad, and the ugly" mainly focused on tools used in agile projects. It focused on gathering statistics on company structure and maturity of agile methods using TargetProcess trial versions. Although the paper has published a couple years ago and in recent years, many new tools have captured the market, it is beneficial as a reference to choose most important tools and metrics [6].

In 2013, "8th Annual State of Agile," written by the VersionOne Company includes a normalized and wide distribution of responses of multitude of channels from companies, engineers, scrum masters, product owners and even self-employed engineers. The respondents are from different countries and questions have focused on details such as reasons for adopting Agile, agile techniques used. The main points of the paper are detailed statistics in the agile methods in projects, and the information about adopting agile methods [7].

In 2014,"Agile Tools Evaluator Guide" written by the VersionOne Company intended to help organizations in choosing software to support their agile teams and processes.

Some of the mentioned research is considerable due to their direct relevance to our research problem. Some of them are sponsored by vendors, therefore the questions probably have been prepared based on the product features of the company. None of the papers and surveys provide comprehensive method and opportunity to select a tool among a wide range of agile tools. Also, none of them provide a methodology for right tool selection regarding the size and maturity of projects and companies for instance for a small startup or large organization.

\section{METHODOLOGY}

Companies that are successful in agile software development know that "Individuals and Interactions" are more important than "Processes and Tools"; but the right agile tools really can affect the enterprise, especially when interactions can be more productive. Thus, how top agile tools are provided and which important factors are essential, are discussed in this section.

\section{A. Tools}

To prepare a list of the best tools in the market, firstly, more than 300 blogs, web pages, including reviews, tutorials and online books have been read. Afterward, we reviewed papers, surveys, and white papers, especially those which had been published in recent years. Secondly, more than 40 graduate students, including $10 \mathrm{PhD}$ students from the computer science department of Florida International University (FIU) during a semester were supposed to choose one or two tools and make a practical research in the enterprise or project using chosen tools and finally they made a video describing tool and their features. Some of them are still adopting agile tools in self-employed projects, senior projects, startups, and even organizations and they provide us precise feedbacks. Also, they installed tools, paid if necessary, and released their results on Github and YouTube [8].

There are different types of management tools. Traditional Project Management Tools, Spreadsheets, Physical Walls and Paper, and commercial modern Agile Project Management Tools. Other than physical tools we consider features like the size of the project, the size of the team, stability of the requirements and complexity of the software for a wide range of available tools to maintain diversity among them. Afterwards the tools are divided into Proprietary tools vs. open source tools. To keep a better comparison, we consider some of the criteria as well like satisfactory aspects of the tools like:

\section{- $\quad$ Ease of Use \\ - Integration with Other Systems \\ - Availability of Reports \\ - Price \\ - Customizability}

\section{B. Criteria to consider}

To prepare a list of the most important criteria to satisfy agile techniques employed, all of the recent surveys were considered. In addition, some feedbacks provided by students helped us to balance some of the vendor's surveys. Six different core criteria definition is presented as follows.

\section{1) Flexibility}

Organizations and companies are different and unique. The agile project management tool should have flexibility to adapt to those differences.

2) Ease of Use

Ease of use is that users can utilize the agile tool without a lot of training and time consuming procedures.

\section{3) Category}

Companies are placed into the categories that fit with their organization's needs. For example, if it is an organization with a hundred users, it is probably not going to want a simple standalone solution.

\section{4) Pricing}

Pricing and cost models are an important factor in any purchase and agile project management tool.

\section{5) Responsiveness}

How responsive are the vendors? How do the vendors support their customers? Responsiveness is how the vendors respond to the needs of their customers.

\section{6) Features}

Features are an essential part of any agile tool evaluation. After you figure out which specifications and features a system supports, understand how those features would be used to perform your project process. 


\section{7) Open source tools}

Agile project management are divided to proprietary tools and Open Source Tools. Open source agile tools may have some restriction while using some features; thus each organization should consider its situation before choosing a tool. Particular features may be vital for one enterprise whereas is not important for another company. The following factors are considerable using open source tools.

- Feature sets

- Usability

- Viability

- Suitability for large companies, projects and products

\section{RESULTS}

The comparison table "Table I, II" is used to compare best agile project management tools. The purpose of the comparison table is to highlight the requirements for which you are looking, and to be able to compare different agile tools against those requirements. Some of the most important key factors should be considered in order to select an agile tool for project management.

\section{A. Life Cycle Management using One Agile Tool}

Storing project information in different multiple tools causes inaccurate results and prevents to comfortable real-time visibility.

\section{B. Cross-Functional Teams}

It means to manage the requirements of the customers, programmers, testers, product owners, and other stakeholders in an integrated environment to enhance collaboration and consistency.

\section{Enterprise Scale}

In order to deployment of an enterprise, agile tools should be able to handle the project structure, tasks, defects and tests.

Table I: Evaluation criteria

\begin{tabular}{|l|l|}
\hline Lifecycle Coverage & $\begin{array}{l}\text { Product and their release. Iteration planning and its tracking, Strategic Goals, backlog and the repository for defects, Test } \\
\text { management }\end{array}$ \\
\hline Simplicity \& Ease of Use & $\begin{array}{l}\text { Customizable dashboards for tracking, Drag and Drop; Shortcut options for actions such as: Close, open and delete; } \\
\text { Interactive environment supporting the daily activities of teams }\end{array}$ \\
\hline Collaboration & $\begin{array}{l}\text { Communication media for teams; Mobile Stream to keep projects moving; Email notifications and RSS feeds; Reporting } \\
\text { and tracking for distributed team members; Customizable boards and coding }\end{array}$ \\
\hline $\begin{array}{l}\text { Analytics, Visibility and } \\
\text { Reporting }\end{array}$ & $\begin{array}{l}\text { Dashboards with sufficient metrics; Advanced planning e.g. what-if analysis; Reports, charts and graphs; Hierarchy } \\
\text { charts, Relationship mapping, Release dependency visibility }\end{array}$ \\
\hline Workspace and Process & $\begin{array}{l}\text { Drag and drop story, task and boards; Customizable methodologies (XP, Scrum, Kanban, etc.); Extensive options for } \\
\text { boards, fields; Color coded visual representation }\end{array}$ \\
\hline Program Management & Release rollouts; Program-level Epicboards; Epic planning; Cross- team planning, tracking \\
\hline $\begin{array}{l}\text { Deployment, Integrity and } \\
\text { Security }\end{array}$ & $\begin{array}{l}\text { Free trial software available; Maturity size-based product versions; Web services API; Project-level security; Integrates } \\
\text { with Existing Tools like Source control systems (e.g. GitHub), bugtrackers (e.g. JIRA). }\end{array}$ \\
\hline
\end{tabular}

Table II: Agile tools comparison chart (A: Full support, B: Quite good, C: Bad, *: Free applicable trial)

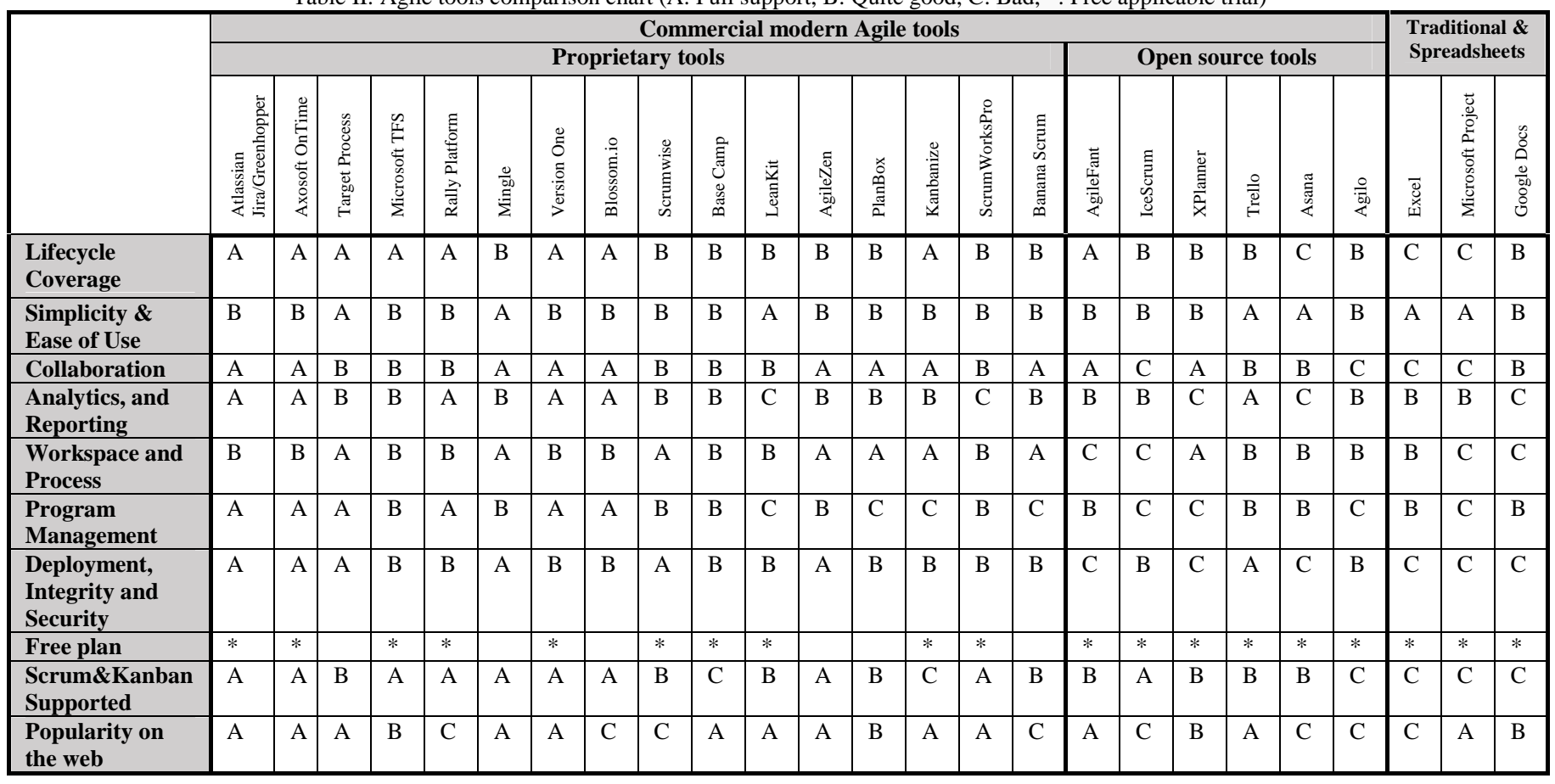




\section{ClassifiCATION}

The comparison tables focus only on top 25 agile project management tools and compare them; but there are also a lot of commercial vendors offering solutions in this market. How to select right agile project management tool for different maturity level? Agile only fits in some company scales and the sad truth is that agile doesn't fit all company scales. So, many agile adoptions in progress right now are going to fail for this reason "Table III".
- $\quad$ Start-up: During this time manager usually struggles to survive.

- Growth stage: Company has added customers and increased sales to new markets and also new professional staff must be added.

- Maturity stage: The business is operating well, with an established market share "Fig. 1 " [9, 10].

Table III: Ease of Use for Different levels of Maturity (A: Quite appropriate, B: good, C: Bad)

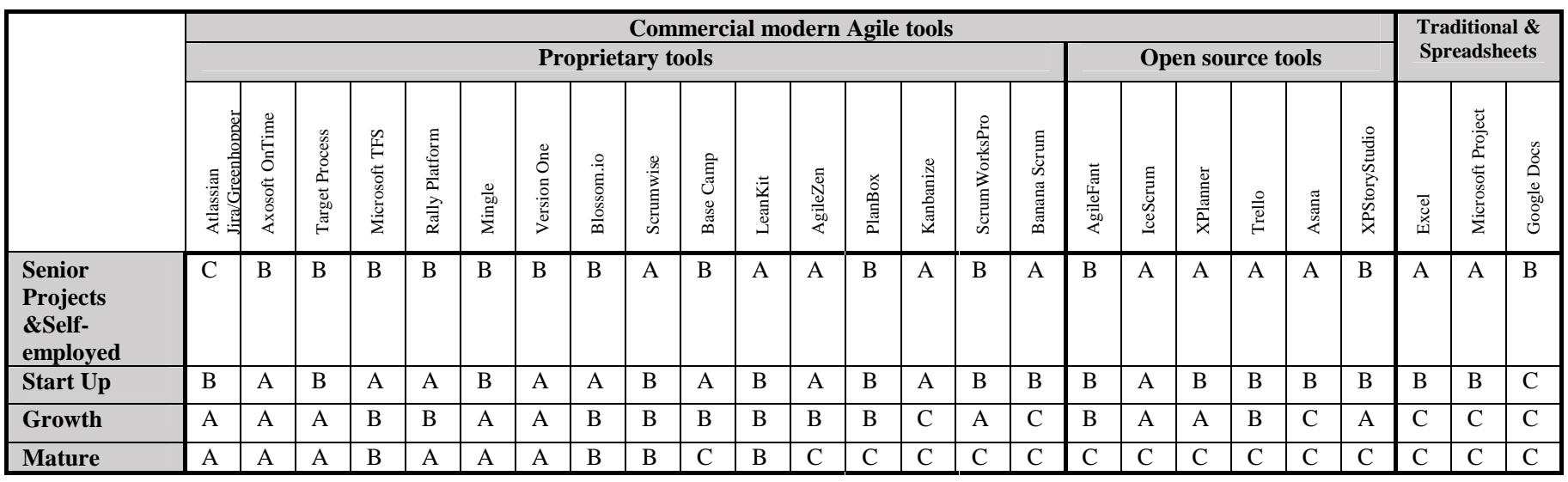

\section{CONCLUSION}

As project team members in the company continue to use agile and enterprise scales agile development within their companies, the challenges of managing different groups continue to increase. Agile software development tools provide solutions to manage this sophisticated process using a framework to maximize the consistency and success of agile development. In this paper, we presented a feature-based classification approach to select best and the right tools. In brief some key factors in this classification reply to these considerable questions:

1. Flexibility: Can the system adapt to how your organization does business? 2. Ease of Use: Will your people be able to use the tool without a couple of hours training? 3. Category: Into which classification of agile project management tools does it fit, and does that class match with the needs of your organization? 4. Responsiveness: How responsive is the organization? 5. Pricing: Does the pricing of the system match the value you will receive? 6. Features: Does the system have enough features to meet your current and future objectives [11]?

Then we classified them in a table based on comprehensive factors:

- Feature-driven Development: Some companies attempt to use a traditional tool that causes their project to be more complicated due to these tools don't support basic agile practices [12].
- Lifecycle Management: Storing project information in different multiple tools causes inaccurate results and prevents to comfortable real-time visibility [13].

- Cross-Functionality: It means to manage the requirements of the customers, programmers, testers, product owners, and other stakeholders in an integrated environment to enhance collaboration and consistency [14].

- Configuration with Flexibility: An agile management tool should let companies to organize, and plan according to their requirements.

- Simplicity: Like agile software project development, the simple one with ease of use is better, but the level of maturity is considerable.

- Enterprise Scale: In order to deployment of an enterprise, agile tools should be able to handle the project structure, tasks, defects and tests [15].

Finally, we classified results in a table and presented a model to select right agile tool based on features of agile software development tool and enterprise needs [17, 18, 19]. In this model, 3 key criteria is applied, 1- cloud ability which indirectly covers security, 2- Open source vs. proprietary, 3- Co-located teams vs. Distributed teams. Finally, at each leaf, a couple of agile tools which best fits in this situation is offered. Due to most of the tools even those which needs high security, have cloud and web based capabilities, our model is based on cloud. 


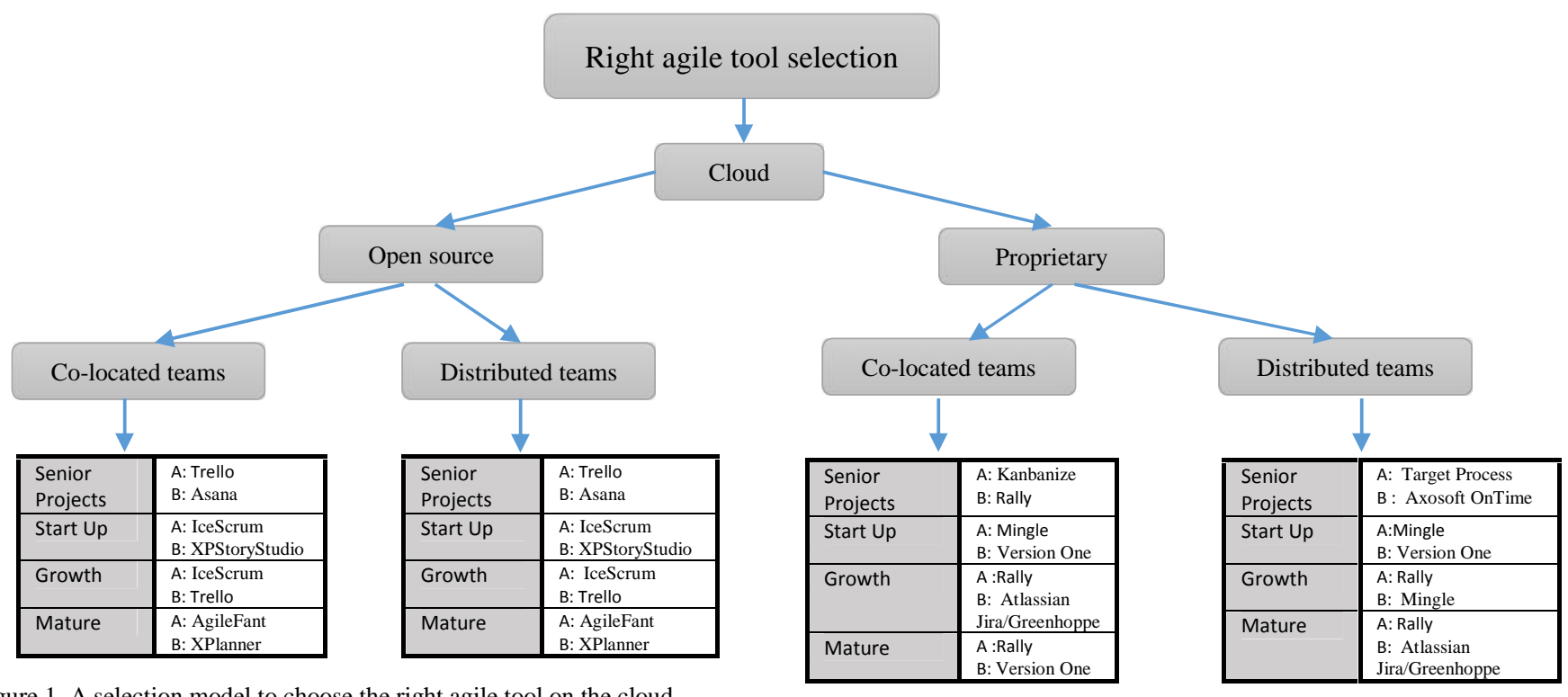

Figure 1. A selection model to choose the right agile tool on the cloud

\section{ACKNOWLEDGMENT}

This research was partly supported by the Leona M. and Harry B. Helmsley Charitable Trust via the Georgia Tech's Vertically Integrated Projects (VIP) Program. The authors also thank the FIU graduate students attended Dr. Sadjadi's Fall 2014 Advanced Software Engineering class for their technical assistance and insightful discussions during the class sessions. This material is based in part upon work supported by the National Science Foundation under Grant Numbers of I/UCRC IIP-1338922, AIR IIP-1237818, SBIR IIP-1330943, III-Large IIS-1213026, MRI CNS-1429345, MRI CNS-0821345, MRI CNS-1126619, CREST HRD-0833093, I/UCRC IIP-0829576, and MRI CNS-0959985.

\section{REFERENCES}

[1] Martin, Robert Cecil. Agile software development: principles, patterns, and practices. Prentice Hall PTR, 2003.

[2] Abrahamsson, Pekka. Agile Software Development Methods: Review and Analysis (VTT publications). 2002.

[3] Azizyan, Gayane, Miganoush Katrin Magarian, and Mira Kajko-Matsson. "Survey of agile tool usage and needs." Agile Conference (AGILE), 2011. IEEE, 2011.

[4] Azizyan, Gayane, Miganoush Magarian, and Mira Kajko-Mattsson. "The Dilemma of Tool Selection for Agile Project Management." ICSEA 2012, The Seventh International Conference on Software Engineering Advances. 2012.

[5] Behrens, Peter. "Agile Project Management (APM) tooling survey results." Trail Ridge consulting (2006).

[6] Dubakov, Michael, and Peter Stevens. "Agile Tools: The Good, the Bad and the Ugly." Report, TargetProcess, Inc (2008).

[7] VersionOne.com. "The 8th Annual“State of Agile" Survey."

[8] https://www.youtube.com/channel/UCucIZz2RRY6tYcKuTDxO8pw

[9] Kumar, Misha, Laszlo Huber, and Milan M. Jovanovic. "Start-up procedure for three-phase six-switch boost PFC rectifier." Applied Power Electronics Conference and Exposition (APEC), 2014 Twenty-Ninth Annual IEEE. IEEE, 2014.

[10] Giardino, Carmine, et al. "What do we know about software development in startups?." Software, IEEE 31.5 (2014): 28-32.

[11] Bustard, David, George Wilkie, and Des Greer. "The maturation of agile software development principles and practice: Observations on successive industrial studies in 2010 and 2012." Engineering of Computer Based Systems (ECBS), 2013 20th IEEE International Conference and Workshops on the. IEEE, 2013.

[12] Thakur, Siddharth, and Harshavardhan Singh. "FDRD: Feature driven reuse development process model." Advanced Communication Control and Computing Technologies (ICACCCT), 2014 International Conference on. IEEE, 2014.

[13] Reichert, Manfred, Alena Hallerbach, and Thomas Bauer. "Lifecycle management of business process variants." Handbook on Business Process Management 1. Springer Berlin Heidelberg, 2015. 251-278.

[14] K Majchrzak, Ann, Philip HB More, and Samer Faraj. "Transcending knowledge differences in cross-functional teams." Organization Science 23.4 (2012): 951-970.

[15] Ambler, Scott W., and Mark Lines. Disciplined agile delivery: A practitioner's guide to agile software delivery in the enterprise. IBM Press, 2012.

[16] Misha, Laszlo Huber, and Milan M. Jovanovic. "Start-up procedure for three-phase six-switch boost PFC rectifier." Applied Power Electronics Conference and Exposition (APEC), 2014 Twenty-Ninth Annual IEEE. IEEE, 2014.

[17] Abrahamsson, Pekka, Nilay Oza, and Mikko T. Siponen. "Agile Software Development Methods: A Comparative Review1." Agile Software Development. Springer Berlin Heidelberg, 2010. 31-59.

[18] Langer, Tomáš, and Pavel Vaněček. "AGILE METHODS IN TECHSTARTUP." IMEA 2012

[19] Turk, Dan, Robert France, and Bernhard Rumpe. "Limitations of agile software processes." arXiv preprint arXiv:1409.6600 (2014). 